\title{
Isovector neutron-proton pairing with particle number projected BCS
}

\author{
N. Sandulescu \\ Institute of Physics and Nuclear Engineering, Post Office Box MG-6, R-76900 Bucharest-Magurele, Romania \\ B. Errea and J. Dukelsky \\ Instituto de Estructura de la Materia, CSIC, Serrano 123, E-28006 Madrid, Spain
}

(Received 24 March 2009; published 29 October 2009)

\begin{abstract}
The particle number projected BCS (PBCS) approximation is tested against the exact solution of the $\mathrm{SO}(5)$ Richardson-Gaudin model for isovector pairing in a system of nondegenerate single-particle orbits. Two isovector PBCS wave functions are considered. One is constructed as a single proton-neutron pair condensate; the other corresponds to a product of a neutron pair condensate and a proton pair condensate. The PBCS equations are solved using a recurrence method and the analysis is performed for systems with an equal number of neutrons and protons distributed in a sequence of equally spaced fourfold (spin-isospin) degenerate levels. The results show that although PBCS offers significant improvement over BCS, the agreement of PBCS with the exact solution is less satisfactory than in the case of the SU(2) Richardson model for pairing between like particles.
\end{abstract}

DOI: 10.1103/PhysRevC.80.044335

PACS number(s): 21.30.Fe, 21.60.Fw

\section{INTRODUCTION}

Neutron-proton $(n p)$ pairing is a longstanding issue in nuclear structure [1,2]. Despite much effort, the specific fingerprints of these correlations in existing nuclear data are not yet clear, nor are the appropriate theoretical tools for their correct treatment. For many years the theoretical framework commonly used to describe the $n p$ pairing correlations was the generalized Hartree-Fock-Bogoliubov (HFB) approach [3]. In this approach the $n p$ pairing, both isovector and isoscalar, is treated simultaneously with neutron-neutron $(n n)$ and protonproton $(p p)$ pairings. However, although the generalized BCS approach treats all types of pairing correlations equally, most of the BCS calculations show that they rarely mix [4]. Thus, in general, there are three BCS solutions that seem to exclude each other: the first, with $n n$ and $p p$ pairs; the second, degenerate to the first in even-even $N=Z$ nuclei with isovector $n p$ pairs; and the third, with isoscalar $n p$ pairs.

Various studies showed that the restoration of particle and isospin symmetries and the inclusion of higher order correlations significantly improved the predictions of the BCS approach for systems with an $n p$ pairing [5-10]. To restore exactly these symmetries, projection operators or projected generator coordinate methods are commonly employed [11]. Less discussed in the literature is an alternative method based on the recurrence relations satisfied by the isovector pairing Hamiltonian averaged on projected BCS (PBCS) wave functions. In this article we implement this method to analyze the dependence of isovector pairing correlations on particle number conservation. As trial eigenstates we use two PBCS condensates, one formed by isovector $n p$ pairs and another by $n n$ and $p p$ pairs. In contrast to the BCS approximation for a system of an even number of pairs, the PBCS solutions corresponding to these two pair condensates are not degenerate. To analyze how much these PBCS solutions offer an improvement over the generalized BCS approach we use the exactly solvable $\mathrm{SO}(5)$ Richardson-Gaudin pairing model [12]. Several previous studies were carried out in the one-level degenerate $\mathrm{SO}(5)$ model [13]. These studies clarified the limitations of the BCS approximation, and the corresponding extensions, by taking into account pair fluctuations in the random phase approximation (RPA) formalism or using boson expansion theories $[6,9,14]$. Studies on number and isospin projection on the isovector pairing Hamiltonian with nondegenerate single-particle levels were reported in Ref. [5]. However, these studies were tested against a solution proposed by Richardson [15] and, later on, shown to be incorrect for systems with more than two pairs [16]. The exact solution of the nondegenerate isovector pairing Hamiltonian was given by Links et al. [17] and afterward generalized to seniority nonzero states, arbitrary degeneracies, and symmetry-breaking Hamiltonians in Ref. [12]. This solution is used here as a benchmark to test the accuracy of PBCS approximations in describing the isovector pairing correlations.

\section{FORMALISM}

We consider an isovector $(T=1)$ pairing Hamiltonian with a constant pairing strength

$$
H=\sum_{i m \tau} \varepsilon_{j_{i}} a_{j_{i} m \tau}^{\dagger} a_{j_{i} m \tau}-g \sum_{i, i^{\prime}, \tau} \hat{j_{i}} \hat{j_{i^{\prime}}} P_{j_{i} \tau}^{+} P_{j_{i^{\prime}} \tau},
$$

where $P_{j_{i}}^{+}=\frac{1}{\sqrt{2}}\left[a_{j_{i}}^{+} a_{j_{i}}^{+}\right]_{0 \tau}^{01}$ is the isovector pair creation operator and $\hat{j}=\sqrt{(j+1 / 2)}$. The first column in the couplings refers to total angular momentum and the second column to total isospin. The Hamiltonian (1) is a particular example of the exactly solvable SO(5) Richardson-Gaudin integrable models. It is exactly solvable for arbitrary single-particle energies $\varepsilon_{j_{i}}$ and pair degeneracies $j_{i}+1 / 2$. The exact solution of this class of Hamiltonians was given in Ref. [12]. Here we treat a simplified version for a system of $L$-equidistant single-particle levels of pair degeneracy 1 , that is, $j_{i}=1 / 2$. The exact solution 
for this system is used as a test for the PBCS approximation with isovector pairing. For comparison, we also show the results of the BCS approximation. The BCS approximation for isovector pairing was discussed in many articles (e.g., Refs. [2,3,18,19]). For the particular case of $N=Z$ systems with the protons and neutrons filling the same shells it can be shown (see Appendix) that there are two BCS solutions: (A) a BCS solution with a nonzero proton-neutron gap, $\Delta_{n p}=$ $\Delta$, and zero gaps for neutron-neutron and proton-proton pairs, $\Delta_{n}=\Delta_{p}=0$; and (B) a BCS solution with $\Delta_{n}=\Delta_{p}=\Delta$ and $\Delta_{p n}=0$. These solutions are degenerate in energy and do not mix with each other. To get the mixing, shown by the exact solution, it is necessary to go beyond BCS (e.g., by restoring isospin conservation $[5,7])$.

In the next section we present the PBCS approximations corresponding to the two degenerate BCS solutions (A) and (B). The PBCS equations are given in the form of recurrence relations and can be applied to general (density-independent) isovector pairing interactions, irrespective of whether they are integrable or not.

\section{A. PBCS approximation with isovector proton-neutron pairs}

We first consider a PBCS wave function corresponding to the solution (A) formed by $N$ isovector neutron-proton pairs. It has the following form:

$$
|N\rangle=\frac{1}{N !}\left(\Gamma_{0}^{+}\right)^{N}|0\rangle,
$$

where $\Gamma_{0}^{+}$is the collective neutron-proton pair operator

$$
\Gamma_{0}^{+}=\sum_{i=1}^{L} x_{i} P_{i 0}^{+} .
$$

This wave function is not normalized and the factor in front is chosen to simplify the form of PBCS equations. The mixing amplitudes $x_{i}$ are determined by minimizing the energy functional

$$
E(x)=\frac{\langle N|H| N\rangle}{\langle N \mid N\rangle} .
$$

The norm and the expectation value of the Hamiltonian are calculated by using recurrence relations. Thus, it can be shown that the norm of the wave function (2) satisfies the equation

$$
\begin{aligned}
\langle N \mid N\rangle= & \frac{1}{N} \sum_{i} x_{i}^{2}\langle N-1 \mid N-1\rangle \\
& -\frac{1}{2 N} \sum_{i} x_{i}^{3}\left\langle N-1\left|P_{i 0}^{+}\right| N-2\right\rangle,
\end{aligned}
$$

where

$$
\left\langle N\left|P_{i 0}^{+}\right| N-1\right\rangle=x_{i}\langle N-1 \mid N-1\rangle-\frac{1}{2} x_{i}^{2}\left\langle N-1\left|P_{i 0}^{+}\right| N-2\right\rangle .
$$

To get the norm to correspond to the system with $N$ protonneutron pairs, the preceding equations must be iterated starting with $\langle 1 \mid 1\rangle=\sum_{i} x_{i}^{2}$ and $\left\langle 1\left|P_{i 0}^{+}\right| 0\right\rangle=x_{i}$.

The expectation values of the particle number operators $N_{i}$, which give the occupation probabilities of the single-particle levels, can be calculated from the equation

$$
\left\langle N\left|N_{i}\right| N\right\rangle=2 x_{i}\left\langle N\left|P_{i 0}^{+}\right| N-1\right\rangle,
$$

where the matrix elements on the right-hand side are given by Eq. (6).

Finally, the matrix elements of the pairing force are given by the equations

$$
\begin{aligned}
\langle N| & P_{i 0}^{+} P_{j 0}|N\rangle \\
= & \frac{1}{4} x_{i}^{2} x_{j}^{2}\left\langle N-2\left|P_{j 0}^{+} P_{i 0}\right| N-2\right\rangle+x_{j}\left\langle N\left|P_{i 0}^{+}\right| N-1\right\rangle \\
& \quad-\frac{1}{2} x_{j}^{2} x_{i}\left\langle N-1\left|P_{j 0}^{+}\right| N-2\right\rangle \\
& \quad+\delta_{i j} \frac{x_{i}^{4}}{4}\left[\langle N-2 \mid N-2\rangle-\frac{1}{2}\left\langle N-2\left|N_{i}\right| N-2\right\rangle\right] \\
\langle N| & P_{i 1}^{+} P_{j 1}+P_{i-1}^{+} P_{j-1}|N\rangle \\
= & \frac{x_{i}^{2} x_{j}^{2}}{4}\left\langle N-2\left|P_{i 1}^{+} P_{j 1}+P_{j-1}^{+} P_{i-1}\right| N-2\right\rangle \\
& +\delta_{i j} \frac{x_{i}^{4}}{2}\left[\langle N-2|| N-2\rangle-\frac{1}{2}\left\langle N-2\left|N_{i}\right| N-2\right\rangle\right] .
\end{aligned}
$$

These equations are iterated starting from $\left\langle 1\left|P_{i 0}^{+} P_{j 0}\right| 1\right\rangle=x_{i} x_{j}$ and $\left\langle 1\left|P_{i 1}^{+} P_{j 1}+P_{i-1}^{+} P_{j-1}\right| 1\right\rangle=0$.

\section{B. PBCS approximation with proton-proton and neutron-neutron pairs}

We now consider a PBCS wave function corresponding to the BCS solution (B) given by the product of two condensates formed by $n n$ and $p p$ pairs. This trial eigenstate has the form

$$
|M M\rangle \equiv|M\rangle \otimes|M\rangle=\frac{1}{(M !)^{2}}\left(\Gamma_{n}^{+} \Gamma_{p}^{+}\right)^{M}|0\rangle,
$$

where $M$ denotes the number of $n n$ and $p p$ pairs, $M=N / 2$, and $\Gamma_{n}^{+}$and $\Gamma_{p}^{+}$are the collective pair operators for neutrons and protons [see Eq. (9)]. As defined here, the wave function (8) is well suited for even-even nuclei. For odd-odd nuclei, the corresponding wave function is formed by $M=(N-1) / 2$ neutron-neutron and proton-proton pairs plus two unpaired nucleons that block the corresponding levels affecting the pairing correlations.

Since the Hamiltonian (1) is symmetric in isospin, for $N=$ $Z$ systems the collective proton and neutron pair operators must have the same mixing amplitudes, that is,

$$
\Gamma_{n}^{+}=\sum_{i=1}^{L} y_{i} P_{i 1}^{+}, \quad \Gamma_{p}^{+}=\sum_{i=1}^{L} y_{i} P_{i-1}^{+}
$$

For the same reason, the norms for the neutron and proton wave functions and the matrix elements for the neutronneutron and proton-proton interaction must satisfy similar recurrence relations. Therefore, in the following we give only the recurrence relations for one kind of particle, neutrons. Thus, the norm of the neutron state $|M\rangle$ and the average 
neutron number are given by

$$
\begin{aligned}
\langle M \mid M\rangle= & \frac{1}{M} \sum_{i} y_{i}^{2}\langle M-1 \mid M-1\rangle \\
& -\frac{1}{M} \sum_{i} y_{i}^{3}\left\langle M-1\left|P_{i 1}^{+}\right| M-2\right\rangle, \\
\left\langle M\left|N_{i}\right| M\right\rangle= & 2 y_{i}\left\langle M\left|P_{i 1}^{+}\right| M-1\right\rangle,
\end{aligned}
$$

where

$$
\left\langle M\left|P_{i 1}^{+}\right| M-1\right\rangle=y_{i}\langle M-1 \mid M-1\rangle-y_{i}^{2}\left\langle M-1\left|P_{i 1}^{+}\right| M-2\right\rangle .
$$

The matrix elements of the neutron-neutron pairing interaction are given by the equations

$$
\begin{aligned}
\left\langle M\left|P_{i 1}^{+} P_{j 1}\right| M\right\rangle= & y_{i}^{2} y_{j}^{2}\left\langle M-2\left|P_{j 1}^{+} P_{i 1}\right| M-2\right\rangle \\
& +y_{j}\left\langle M\left|P_{i 1}^{+}\right| M-1\right\rangle \\
& -y_{j}^{2} y_{i}\left\langle M-1\left|P_{j 1}^{+}\right| M-2\right\rangle \\
& +\delta_{i j} y_{i}^{4}\left\langle M-2\left|1-N_{i}\right| M-2\right\rangle .
\end{aligned}
$$

The iterations are started with the matrix elements $\left\langle 1\left|P_{i 1}^{+} P_{j 1}\right| 1\right\rangle=y_{i} y_{j}$. Equations (10) through (13) are very simple and can be used as an alternative to the projecting operator method commonly applied for systems with likeparticle pairing [20].

The matrix elements of the $T=1$ proton-neutron interaction involve the total wave function $|M M\rangle$. They are given by the recurrence relation

$$
\begin{aligned}
& \left\langle M M\left|P_{i 0}^{+} P_{j 0}\right| M M\right\rangle \\
& \quad=y_{i}^{2} y_{j}^{2}\left\langle M-1 M-1\left|P_{i 0}^{+} P_{j 0}\right| M-1 M-1\right\rangle \\
& \quad+\delta_{i j} x_{i}^{4}\langle M-1 \mid M-1\rangle\left\langle M-1\left|1-N_{i}\right| M-1\right\rangle .
\end{aligned}
$$

The starting matrix elements are $\left\langle 11\left|P_{i 0}^{+} P_{j 0}\right| 11\right\rangle=\delta_{i j} x_{i}^{4}$. As can be seen from the previous equations, the recurrence relations for the PBCS wave functions (2) and (8) are very similar and easy to implement in numerical calculations.

\section{RESULTS AND DISCUSSION}

The results presented in this section correspond to a sequence of $L$ equally spaced fourfold degenerate levels (total angular momentum $j=1 / 2$ ) with single-particle energies $\varepsilon_{i}=(i-1) / 2, \quad i=1,2, \ldots, L$, and filled with $N=L / 2$ proton-neutron pairs (quarter filling). We consider systems with $N=2$ to $N=12$ pairs, which correspond to typical sizes of open shell $N=Z$ nuclei. The strength of the pairing interaction is varied to cover all regimes from weak to strong coupling. For these systems we test the accuracy of the PBCS approximations by comparing correlation energies, odd-even mass differences, and occupation probabilities against the exact solution. To complete the accuracy test it is useful to also analyze the overlap between the PBCS and the exact wave functions. However, due to the complexity of the exact solution [12], the derivation of the explicit forms of the overlaps is a difficult problem that has not yet been solved.

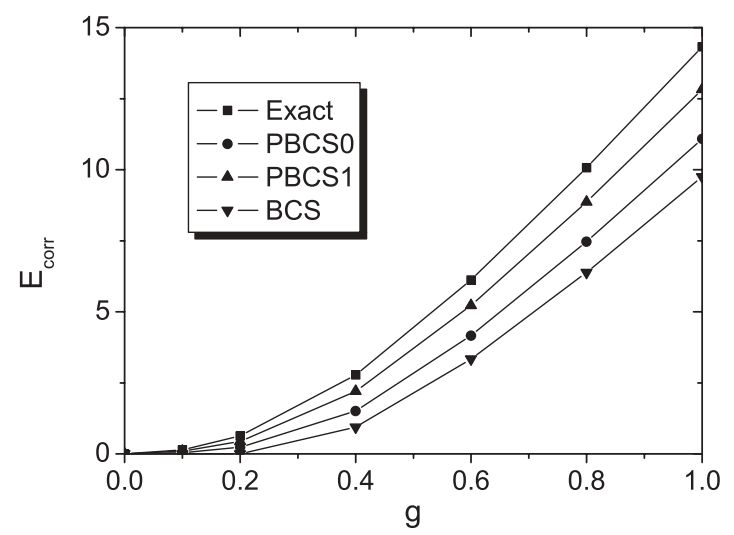

FIG. 1. Correlation energy for four $p n$ pairs.

We start the analysis by focusing on correlation energies. They are defined as

$$
E_{\text {corr }}(g)=E_{\text {nor }}(g)-E(g),
$$

where $E_{\text {nor }}$ and $E(g)$ are the ground-state energies of the system in the normal and the correlated phase, respectively. Some representative results are shown in Figs. 1 through 3. All energies are given in units of the single-particle level spacing. In these figures, $\mathrm{PBC} 0$ corresponds to the variational wave function (2) of $T_{z}=0 n p$ pairs and PBCS1 corresponds to the variational wave function $(8)$ of $n n\left(T_{z}=1\right)$ and $p p$ $\left(T_{z}=-1\right)$ pairs. The two BCS solutions corresponding to these two types of pairs are called BCS0 and BCS1. In even-even systems these two BCS solutions are degenerate and are simply called BCS. Particle number projection breaks this degeneracy.

As can be see in Figs. 1 and 2, both PBCS solutions perform better than BCS for even systems, with PBCS1 capturing more correlations and lowering the ground-state energy. However, as shown in Fig. 3, for a system with an odd number of pairs the lowest energy solution is PBCSO. It can also be seen that, as a result of the blocking, the systems with an odd number of pairs in the solution PBCS1 become even higher in energy than the BCS solution.

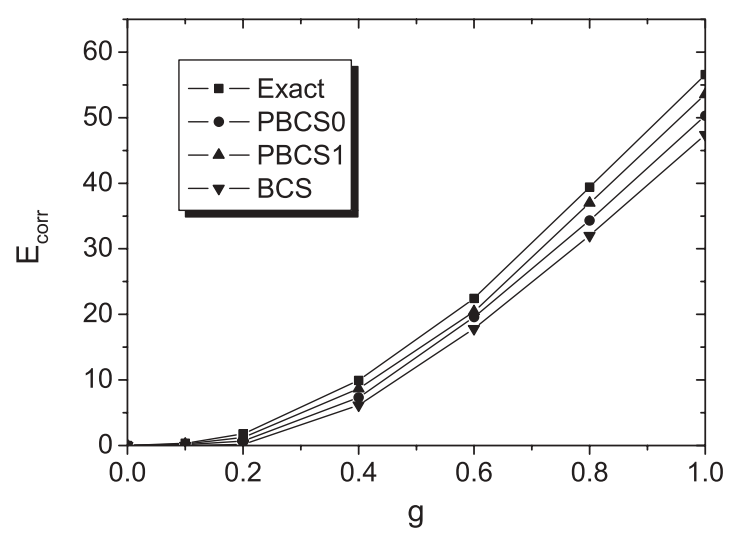

FIG. 2. Correlation energy for eight $p n$ pairs. 


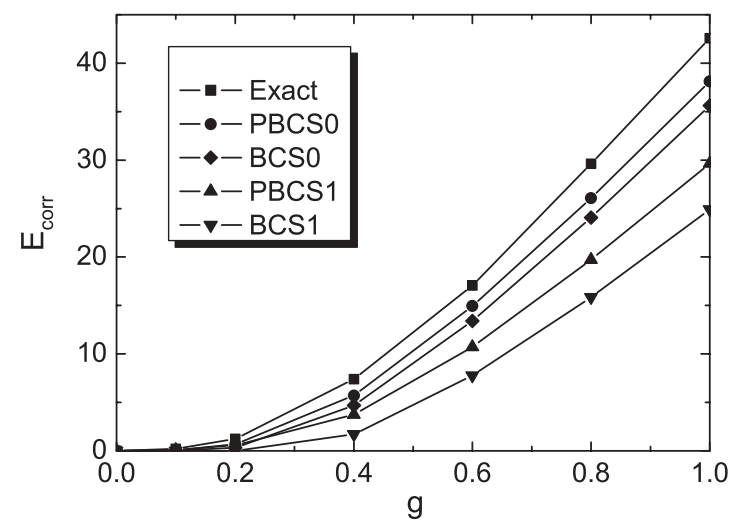

FIG. 3. Correlation energy for seven $p n$ pairs.

The errors relative to the exact results are shown in Fig. 4. It can be seen that, although PBCS gives better results than BCS, the errors remain significant. The reason for this is that the PBCS functions (2) and (8) do not properly take into account the pairing interaction among the pairs with a $T_{z}$ different from what is considered in the trial eigenstate. For example, consider the systems with eight and seven pairs and the interaction strength $g=0.4$. In the system with eight pairs, the wave function PBCS1 gives an energy of -25.71 for the $T_{z}= \pm 1$ part of the Hamiltonian compared to -0.84 for the $T_{z}=0$ part. The situation is opposite for the system with seven pairs; in this case the wave function PBCSO gives an interaction energy of -18.04 for the $T_{z}=0$ component compared to about -1.42 for $T_{z}= \pm 1$.

Another quantity we analyze is the odd-even mass difference along the $N=Z$ line defined as

$$
\Delta^{(3)}(M)=\frac{1}{2}[2 E(M+1)-E(M)-E(M+2)] .
$$

Figure 5 shows the odd-even mass difference for a system with $M=8 p n$ pairs as a function of interaction strength. It can be seen that the PBCS results start to deviate significantly from the exact values when the interaction becomes stronger. The manner in which the odd-even mass difference depends on the number of pairs is depicted in Fig. 6. As expected, the

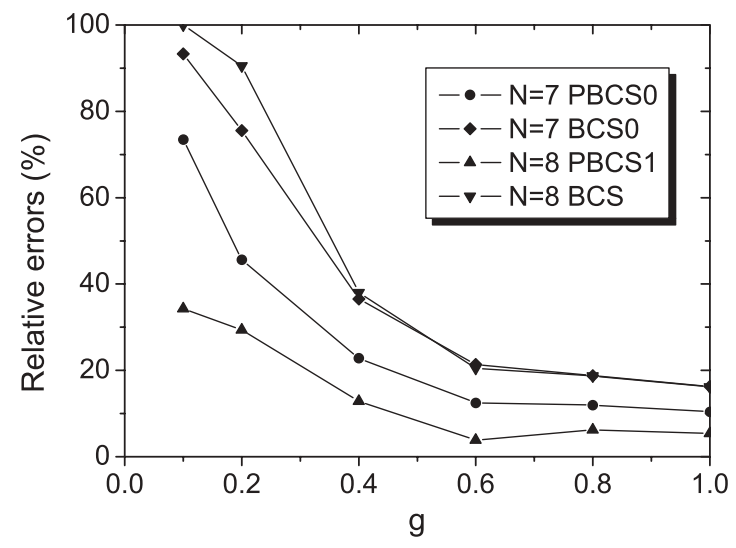

FIG. 4. Errors of the correlation energies in systems with seven and eight $p n$ pairs.

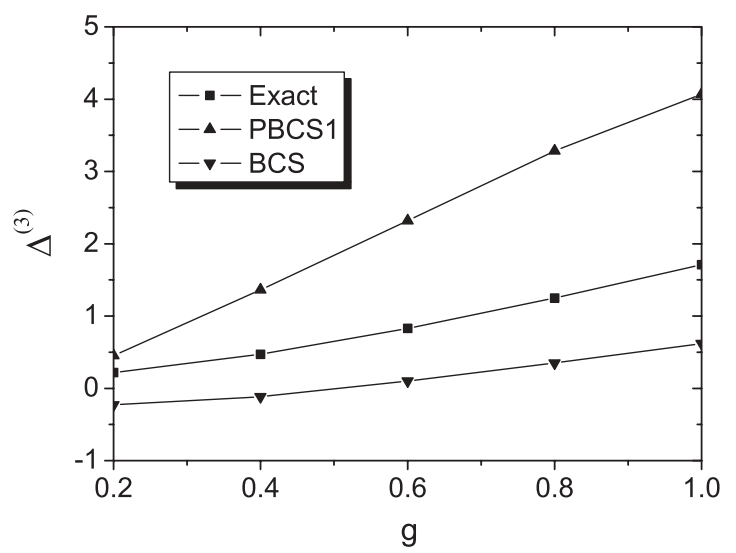

FIG. 5. Odd-even mass difference for a system with eight $p n$ pairs.

BCS results do not show the staggering exhibited by the exact solution. The reason for this is that in the BCS the solutions (A) and (B) are degenerate in energy. However, the staggering is present in the PBCS calculations. This is because in going from the even-even to odd-odd systems the ground state is changing from PBCS0 to PBC1, which are not degenerate. As seen in Fig. 6, the shift between the two solutions overestimates the oscillations present in the exact solution. The reason for this is that the errors in odd systems are larger than in even systems (see Fig. 4).

Next we discuss in brief the occupation probabilities corresponding to BCS and PBCS calculations. Figures 7 and 8 show the quantity $\kappa_{i}^{2}=v_{i}^{2}\left(1-v_{i}^{2}\right)$, where $v_{i}^{2}$ is the occupation probability of the orbit $i$. In BCS, $\kappa_{i}$ is the pairing tensor and determines the pair transfer form factor. From Figs. 7 and 8 we see that the PBCS gives results close to the exact solution for both values of the coupling strength. BCS overestimates the value of $\kappa_{i}^{2}$ at the weak coupling $(g=0.25)$ in the region around the Fermi energy, where the pairing correlations are stronger. Conversely, the values of $\kappa_{i}^{2}$ for the states further than an energy interval of the order of the pairing gap are underestimated. These results are similar to the ones obtained in Ref. [21] for like-particle pairing. For stronger interactions ( $g=0.4$ ) BCS gives results closer to the exact solution.

Up until this point, we considered two PBCS solutions, corresponding to the BCS solutions (A) and (B) mentioned

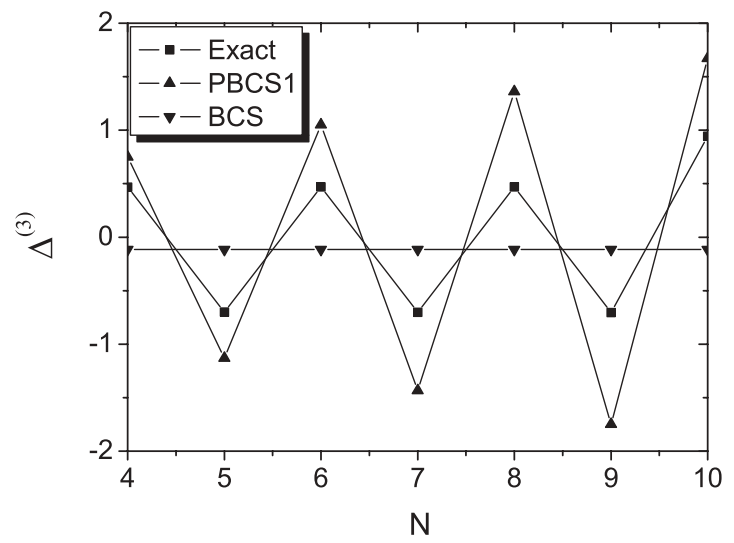

FIG. 6. Odd-even mass difference calculated along $N=Z$ line. 


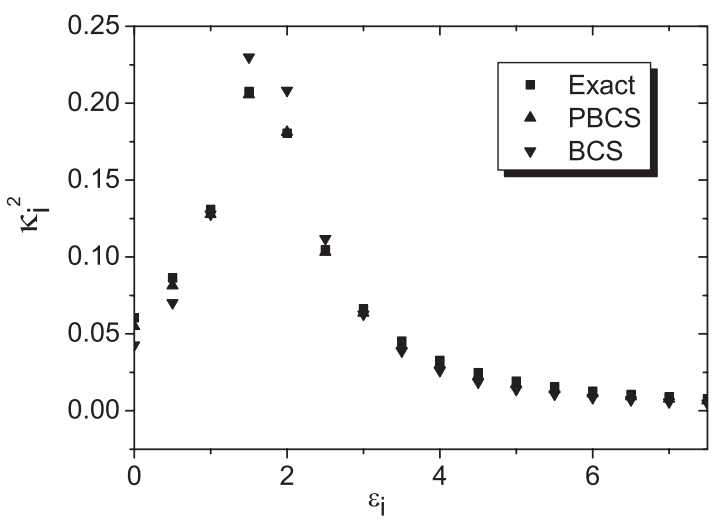

FIG. 7. $\kappa_{i}^{2}=v_{i}^{2}\left(1-v_{i}^{2}\right)$ as a function of the single-particle energies $\varepsilon_{i}$ for a system with eight $p n$ pairs and pairing strength $g=0.25$.

in Sec. II, which do not mix the pn pairing with the pairing between like particles. The issue we now discuss briefly is how one can construct more general particle-conserving trial eigenstates in which $n p$ and like-particles pairing coexist together. Many studies [5,7,9] suggested that to get the coexistence of both types of pairings one needs to restore the isospin conservation. For $N=Z$ systems with an even number of $n p$ pairs, one alternative for an isospin-conserving wave function is $|\Psi\rangle=\left(A^{+}\right)^{(N+Z) / 4}|0\rangle$, where $A^{+}$is an alpha-like operator with the isospin $T=0$, that is,

$$
A^{+} \propto \sum_{i j} z_{i j}\left(P_{i 1}^{+} P_{j-1}^{+}+P_{i-1}^{+} P_{j 1}^{+}-P_{i 0}^{+} P_{j 0}^{+}\right)
$$

Unfortunately, the calculations with such trial eigenstates are very complicated. There is, however, a particular case that can be easily solved by using the two particular solutions PBCS0 and PBCS1. This case corresponds to a system with $N=Z=2$ and an operator $A^{+}$with separable amplitudes, $z_{i j}=z_{i} z_{j}$. The results for this case are shown in Table I. As can be seen, this trial eigenstate, obtained by combining the PBCS0 and PBCS1 solutions into a state of zero isospin, gives practically the exact result for the correlation energy. Whether a similar trial eigenstate can also give a very good

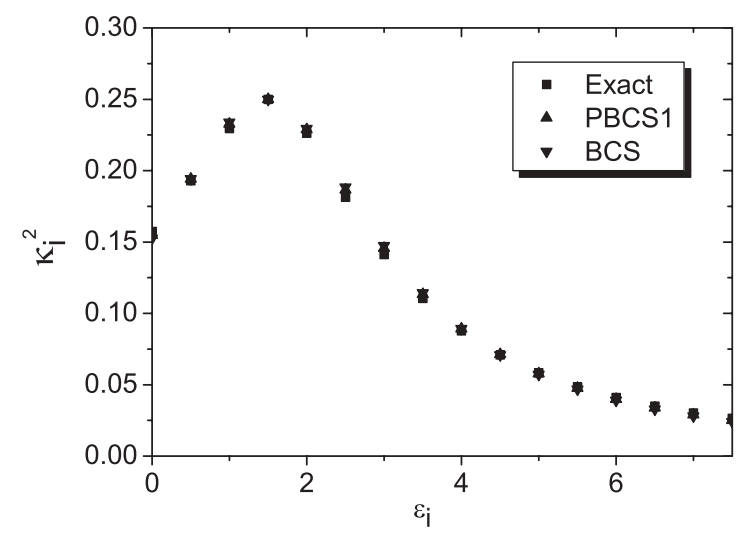

FIG. 8. The same as in Fig. 7 for the pairing strength $g=0.4$.
TABLE I. Correlation energies for a system composed of two isovector $p n$ pairs distributed in four levels with the energies $\epsilon_{i}=(i-1) / 2, i=1,2,3,4$. The binding energies and the pairing strength $g$ are in units equal to the distance between two consecutive single-particle levels.

\begin{tabular}{lcccc}
\hline \hline$g$ & Exact & Pbcs0 + Pbcs1 & Pbcs1 & Pbcs0 \\
\hline 0.1 & 0.05587 & 0.0557 & 0.0376 & 0.0189 \\
0.2 & 0.22006 & 0.2192 & 0.1517 & 0.0779 \\
0.4 & 0.81330 & 0.8114 & 0.5924 & 0.3233 \\
0.6 & 1.64761 & 1.6461 & 1.2551 & 0.7364 \\
0.8 & 2.61989 & 2.6190 & 2.0601 & 1.2972 \\
1.0 & 3.66946 & 3.6689 & 2.9487 & 1.9683 \\
\hline \hline
\end{tabular}

accuracy for systems with more than two $p n$ pairs is not yet clear.

\section{SUMMARY AND CONCLUSIONS}

We analyze the accuracy of PBCS approximation for describing isovector pairing correlations in $N=Z$ systems. The study is done for an exactly solvable Hamiltonian with $\mathrm{SO}(5)$ symmetry. In the PBCS calculations we consider two kinds of trial eigenstates: (1) a condensate of isovector neutronproton pairs and (2) a product of two condensates formed by neutron-neutron and proton-proton pairs. Solution (1) gives the lowest ground-state energy for odd-odd $N=Z$ systems, whereas solution (2) provides the lowest energy for even-even systems. The PBCS approximation gives much better correlation energies than the BCS one and it is able to describe the staggering of odd-even mass difference calculated along the $N=Z$ line. However, compared to the pairing between like particles, for which the PBCS approximation gives results very close to the exact solution of the $\mathrm{SU}(2)$ model [21], the accuracy of the PBCS approximation for isovector pairing is less satisfactory. The reason for this is that the PBCS is unable to treat correctly that part of the isovector force that describes the interaction among the pairs that are not included in the PBCS condensate. Going beyond PBCS will imply including the isospin projection and/or taking into account quartet correlations. We are currently working along the latter direction.

\section{ACKNOWLEDGMENTS}

We thank R. J. Liotta, P. Schuck, and R. Wyss for valuable discussions This work was supported by Romanian PN II under IDEI Grant No. 270 and by the Spanish DGI under Grant No, FIS2006-12783-C03-01. B. E. was supported by CE-CAM.

\section{APPENDIX}

We show in this appendix that the Hamiltonian (1) in the BCS approximation has the two degenerate solutions $(A)$ and $(B)$ of Sec. II for $N=Z$ systems. The proof follows Ref. [18], which contains one of the first discussions on 
isospin invariance for isovector pairing Hamiltonians in the BCS approximation.

As in Ref. [18] we start with a general isovector pairing Hamiltonian written explicitly in the neutron and proton variables, that is,

$$
\begin{aligned}
\hat{H}= & \sum_{j m} \varepsilon_{j}\left(a_{j m}^{+} a_{j_{m}}+b_{j m}^{+} b_{j_{m}}\right) \\
& -\frac{1}{2} \sum_{j, j^{\prime}} G_{j, j^{\prime}} \hat{j} \hat{j}^{\prime}\left\{\left[a_{j}^{+} a_{j}^{+}\right]^{0}\left[a_{j^{\prime}} a_{j^{\prime}}\right]^{0}\right. \\
& \left.+\left[b_{j}^{+} b_{j}^{+}\right]^{0}\left[b_{j^{\prime}} b_{j^{\prime}}\right]^{0}+2\left[a_{j}^{+} b_{j}^{+}\right]^{0}\left[b_{j^{\prime}} a_{j^{\prime}}\right]^{0}\right\},
\end{aligned}
$$

where the operators $a^{+}(a)$ and $b^{+}(b)$ create (annihilate) a neutron or a proton, respectively, and the notation $[\ldots]^{0}$ means coupling to total angular momentum $J=0$. A straightforward alternative to introduce the BCS approximation is to replace this Hamiltonian by the linearized one-body Hamiltonian

$$
\begin{aligned}
\hat{H}_{L}= & \sum_{j m} \varepsilon_{j}\left(a_{j m}^{+} a_{j_{m}}+b_{j m}^{+} b_{j_{m}}\right) \\
& -\frac{1}{2} \sum_{j} \Delta_{n j} \hat{j}\left\{\left[a_{j}^{+} a_{j}^{+}\right]^{0}+\left[a_{j} a_{j}\right]^{0}\right\} \\
& -\frac{1}{2} \sum_{j} \Delta_{p j} \hat{j}\left\{\left[b_{j}^{+} b_{j}^{+}\right]^{0}+\left[b_{j} b_{j}\right]^{0}\right\} \\
& -\sum_{j} \Delta_{n p j} \hat{j}\left\{\left[a_{j}^{+} b_{j}^{+}\right]^{0}+\left[b_{j} a_{j}\right]^{0}\right\},
\end{aligned}
$$

where neutron, proton, and the neutron-proton gaps are given by

$$
\begin{aligned}
\Delta_{n j} & =\sum_{j^{\prime}} G_{j, j^{\prime}} \hat{j}^{\prime}\left\langle\left[a_{j^{\prime}} a_{j^{\prime}}\right]^{0}\right\rangle, \\
\Delta_{p j} & =\sum_{j^{\prime}} G_{j, j^{\prime}} \hat{j}^{\prime}\left\langle\left[b_{j^{\prime}} b_{j^{\prime}}\right]^{0}\right\rangle, \\
\Delta_{n p j} & =\sum_{j^{\prime}} G_{j, j^{\prime}} \hat{j}^{\prime}\left\langle\left[a_{j^{\prime}} b_{j^{\prime}}\right]^{0}\right\rangle .
\end{aligned}
$$

In Ref. [18] it is shown that for a separable interaction, $G_{j, j^{\prime}}=g_{j} g_{j^{\prime}}$, it is possible to find a state-independent rotation in the isospin space that cancels the coupling term in the transformed Hamiltonian. Here we take a particular rotation, convenient for $N=Z$ systems. More precisely, we introduce a new set of operators defined by

$$
\begin{aligned}
& \bar{a}_{j m}=\frac{1}{\sqrt{2}}\left(a_{j m}-b_{j m}\right), \\
& \bar{b}_{j m}=\frac{1}{\sqrt{2}}\left(a_{j m}+b_{j m}\right) .
\end{aligned}
$$

In the new representation the Hamiltonian $H_{L}$ can be written in this same form, but with new gaps given by

$$
\begin{aligned}
& \Delta_{\bar{a} j}=\frac{1}{2}\left(\Delta_{n j}+\Delta_{p j}\right)-\Delta_{n p j}, \\
& \Delta_{\bar{b} j}=\frac{1}{2}\left(\Delta_{n j}+\Delta_{p j}\right)+\Delta_{n p j}, \\
& \Delta_{\bar{a} \bar{b} j}=\frac{1}{2}\left(\Delta_{n j}-\Delta_{p j}\right) .
\end{aligned}
$$

For $N=Z$ systems with the protons and neutrons filling the same shells, $\Delta_{n j}=\Delta_{p j}=\Delta$, which implies that $\Delta_{\bar{a} \bar{b} j}=$ 0 . Consequently, in the new representation the linearized Hamiltonian is a sum of two uncoupled terms, and hence the BCS approximation is reduced to two independent BCS solutions. These solutions must fulfill two constraints. One is for the average number of particles, which is simple to impose because in the new variables,

$$
\hat{N}=\sum_{j m}\left(\bar{a}_{j m}^{+} \bar{a}_{j m}+\bar{b}_{j m}^{+} \bar{b}_{j m}\right) .
$$

The second condition must enforce the conservation, in average, of the isospin. Since in the new variables the isospin operator contains mixed terms in $\bar{a}$ and $\bar{b}$, in Ref. [18] the constraint is put on the operator

$$
\hat{\tau}=\sum_{j m}\left(\bar{a}_{j m}^{+} \bar{a}_{j m}-\bar{b}_{j m}^{+} \bar{b}_{j m}\right) .
$$

It can be shown (see Ref. [18] for details) that (i) the expectation value of $\hat{\tau}$ can be interpreted as the isospin quantum number and (ii) a constraint based on the genuine isospin operator cannot give a lower energy minimum than the minimum obtained with the constraint on $\hat{\tau}$.

For $N=Z$ systems the isospin is zero, so one should impose

$$
\langle\tau\rangle=\sum_{j}(2 j+1)\left(v_{\bar{a} j}^{2}-v_{\bar{b} j}^{2}\right)=0,
$$

where $v_{\bar{a} j}^{2}, v_{\bar{b} j}^{2}$ are the occupation probabilities in the new variables. They have the standard BCS expressions:

$$
v_{\bar{a} j}^{2}=\frac{1}{2}\left(1-\frac{\varepsilon_{j}-\lambda}{\sqrt{\left(\varepsilon_{j}-\lambda\right)^{2}+\Delta_{\bar{a} j}^{2}}}\right) .
$$

One can thus notice that for $N=Z$ systems in which the protons and the neutrons are filling the same shell the condition $\langle\tau\rangle=0$ implies $\Delta_{\bar{a} j}^{2}=\Delta_{\bar{b} j}^{2}$. As can be seen from the expressions of the gaps just given, this condition can be fulfilled in two cases: (A) $\Delta_{n}=\Delta_{p}=\Delta, \Delta_{n p}=0$ and (B) $\Delta_{n}=\Delta_{p}=0, \Delta_{n p}=\Delta$. The two solutions are degenerate because the expectation value of the Hamiltonian depends on the occupation probabilities, which are the same in the two cases.
[1] A. M. Lane, Nuclear Theory (Benjamin, New York, 1964).

[2] A. Goswami, Nucl. Phys. 60, 228 (1964).

[3] A. L. Goodman, in Advances in Nuclear Physics, edited by J. Negele and E. Vogt (Plenum, New York, 1979), Vol. 11, p. 263.

[4] A. L. Goodman, Phys. Rev. C 60, 014311 (1999).
[5] H.-T. Chen, H. Muther, and A. Faessler, Nucl. Phys. A297, 445 (1978).

[6] J. Engel, K. Langanke, and P. Vogel, Phys. Lett. B389, 211 (1996).

[7] J. Engel, S. Pittel, M. Stoitsov, P. Vogel, and J. Dukelsky, Phys. Rev. C 55, 1781 (1997). 
[8] W. Satula and R. Wyss, Phys. Lett. B393, 1 (1997).

[9] J. Dobes and S. Pittel, Phys. Rev. C 57, 688 (1998).

[10] D. S. Delion, J. Dukelsky, P. Schuck, E. J. V. de Passos, and F. Krmpotic, Phys. Rev. C 62, 044311 (2000).

[11] P. Ring and P. Schuck, The Nuclear Many-Body Problem (Springer-Verlag, New York, 1980).

[12] J. Dukelsky, V. G. Gueorguiev, P. Van Isacker, S. Dimitrova, B. Errea, and S. Lerma H., Phys. Rev. Lett. 96, 072503 (2006).

[13] K. T. Hecht, Phys. Rev. 139, B794 (1965).

[14] D. S. Delion, J. Dukelsky, and P. Schuck, Phys. Rev. C 55, 2340 (1997).
[15] R. W. Richardson, Phys. Rev. 144, 874 (1966).

[16] F. Pan and J. P. Draayer, Phys. Rev. C 66, 044314 (2002).

[17] J. Links, H.-Q. Zhou, M. D. Gould, and R. H. McKenzie, J. Phys. A 35, 6459 (2002).

[18] J. N. Ginocchio and J. Weneser, Phys. Rev. 170, 859 (1968).

[19] D. R. Bes, O. Civitarese, E. E. Maqueda, and N. N. Scoccola, Phys. Rev. C 61, 024315 (2000).

[20] K. Dietrich, H. J. Mang, and J. H. Pradal, Phys. Rev. 135, B22 (1964).

[21] N. Sandulescu and G. F. Bertsch, Phys. Rev. C 78, 064318 (2008). 